\title{
Erratum
}

\section{Isolation and characterization of a human homologue of the latrophilin gene from a region of 1p31.1 implicated in breast cancer}

\author{
Gavin RM White, Jenny M Varley and Jim Heighway \\ Oncogene, (1998) 17, 3513-3519.
}

The sequence file used to construct figure 2 of the above manuscript, showing the open reading frame of the $L P H H 1$ transcript, contained three data entry errors. Two bases were incorrect and a run of three nucleotides was omitted. The corrected sequence (Accession no. AJ131581) has alterations to two encoded residues and the insertion of a third. The functional predictions outlined in the manuscript for the various regions of the protein are unchanged by these corrections. However, it should be noted that the open reading frame of all transcripts (except those splice variants that encode truncated peptides) are now three nucleotides longer. The alterations are:
1313
ctt tgc gtg to ctt tac gtg
$\mathrm{C}$ to $\mathrm{Y}$
$3835 \quad$ ggc agc aag to ggc agc agc aag GSK to GSSK
4145 (now 4148) ctt aaa gac to ctt aga gac
$\mathrm{K}$ to $\mathrm{R}$

Nucleotide designations refer to those in figure 2 with amended position for the third alteration in brackets. 